\title{
Chronique de nos « Réflexions » et de nos figurations
}

\section{Thérèse Goyet}

\section{(2) OpenEdition \\ 1 Journals}

Édition électronique

URL : http://journals.openedition.org/ccibp/423

DOI : $10.4000 /$ ccibp.423

ISSN : 2493-7460

Éditeur

Centre international Blaise Pascal

\section{Édition imprimée}

Date de publication : 16 octobre 1984

Pagination : 1-3

ISSN : 0249-6674

\section{Référence électronique}

Thérèse Goyet, "Chronique de nos «Réflexions » et de nos figurations », Courrier du Centre

international Blaise Pascal [En ligne], 6 | 1984, mis en ligne le 27 novembre 2015, consulté le 24

septembre 2020. URL : http://journals.openedition.org/ccibp/423 ; DOI : https://doi.org/10.4000/

ccibp. 423

Ce document a été généré automatiquement le 24 septembre 2020.

Centre international Blaise Pascal 


\section{Chronique de nos « Réflexions » et de nos figurations}

\section{Thérèse Goyet}

L'été 1985 sera probablement écoulé lorsque nos associés recevront cette livraison marquée 1984. Nous tenons la promesse que nous avons faite de leur écrire une fois l'an, (au minimum, car nous avons envoyé divers petits courriers) mais aux apparences de la rigoureuse périodicité nous préférons une continuation concrète. Nous le disions l'an dernier. En gros chacun de nos Courriers récapitule l'année universitaire qui l'a précédé. Or cette dernière année ne se laisse pas facilement clôturer et c'est avec un certain plaisir que nous parlerons des prolongements qui sont encore en train.

8 novembre 1984. - Les congressistes sur le lieu de la maison natale.

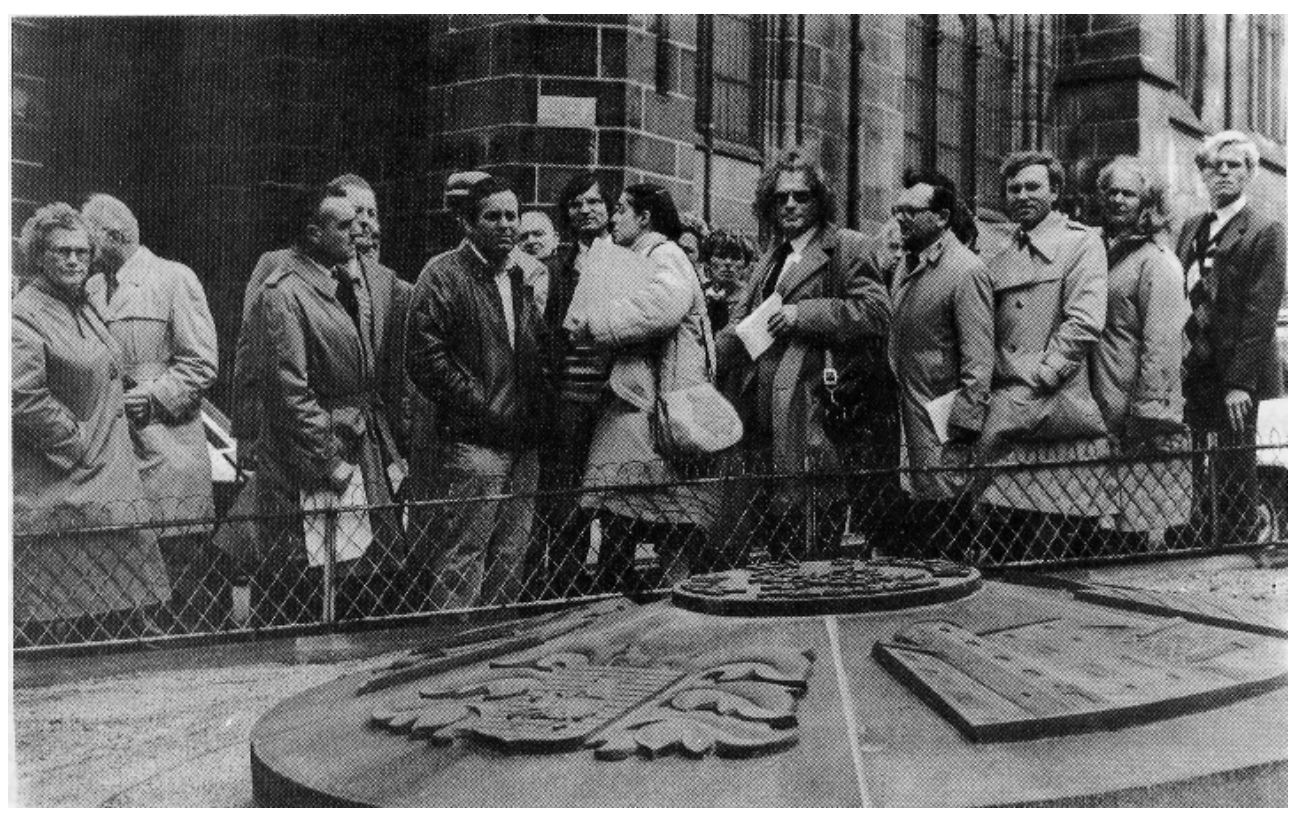

Cliché Roland Jouanisson 
Vraiment le CIBP a été bien occupé, c'est-à-dire, non point diverti par les tracas inhérents au monde contemporain et qui assaillent particulièrement une entreprise en période de croissance, mais adonné à sa vocation qui est « d'accroître... la connaissance et le rayonnement de Blaise Pascal » (article premier de nos statuts). La question des moyens adaptés se pose de façon chaque fois nouvelle, et chaque fois elle se résout empiriquement par le plein vouloir des personnes qui s'engagent. Un projet est suscité, il se précise, il s'actualise, il étend ses rameaux et il enfonce ses racines, grâce aux amitiés - déclarées sur la place ou jusque-là virtuelles - qui la portent et la favorisent comme si elles l'avaient attendu, conçu, désiré.

Donc le projet de « journées de réflexion » consacrées à « l'accès aux Pensées de Pascal » qu'annonçait notre Courrier $n^{\circ} 5$, a été remarquablement bien accueilli. M. le Recteur Jean-Claude Dischamps a tenu à exprimer sa confiance en introduisant nos journées de novembre, et il trouva le temps pour venir conclure avec la même cordialité nos journées de mai qui se trouvaient placées dans la Semaine de l'Éducation. La Mission académique à la Formation des Personnels de l'Éducation nationale a concentré ses efforts en notre faveur et dégagé le plus grand nombre possible de professeurs des classes de première et de terminale pour deux fois un stage de trois jours. Ces absences d'enseignants sont coûteuses à tous les points de vue, mais, a-t-il jamais été facile de découvrir le temps commode pour chaque chose nécessaire? La difficulté, $\mathrm{M}$. l'Inspecteur d'Académie Jacques Marty l'a donc résolue avec une franche volonté d'optimisme. L'Inspection générale était représentée par M. l'Inspecteur Daniel Dubois, fervent pascalien. Le Centre Régional de Documentation Pédagogique (CRDP) accueillant les pédagogues des Pensées les a tout à fait persuadés qu'ils étaient chez lui chez eux. Son excellent équipement audio-visuel a favorisé les diverses techniques d'expression. Nous eûmes, hélas! le regret de n'avoir pas à projeter le film le plus important qui ait été réalisé sur Pascal, celui de Roberto Rossellini, pour lequel nous avions épuisé nos moyens de recherche. La convivialité promise s'instaura d'elle-même au Restaurant universitaire des Cézeaux qui nous donnait, avec de solides plaisirs de table, un espace de liberté dans un cadre presque naturel. La récréation, ce fut aussi la visite, le 8 novembre 1984, de Clermont au temps de Pascal, sous la conduite ferme et gracieuse de $\mathrm{M}^{\text {lles }}$ MarieFrance Cussinet et Marie-Anne Barnier. Découverte pour la plupart d'entre nous car les guides imprimés ignorent la maison de l'Oratoire, si chère à la famille Pascal, et le cloître des anciennes Ursulines et bien d'autres témoins encore. À la seconde session la récréation se fit re-création, les Pensées s'élaborant dans nos mémoires à l'audition de ce récital dont notre ami Descotes nous entretient plus loin. Ici la pédagogie vivante s'investissait corps et âme dans une généreuse fidélité à la lettre du texte inspiré. 


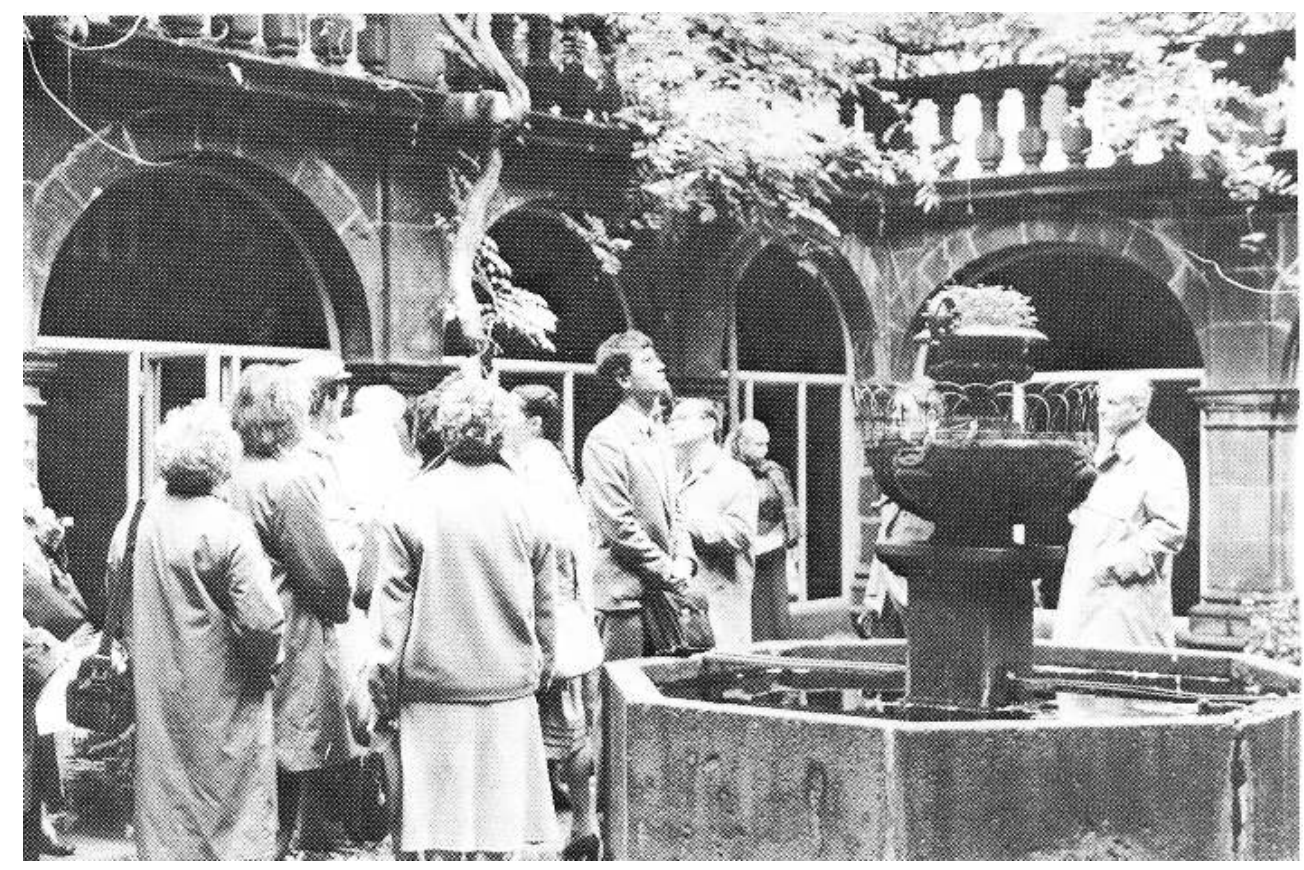

Cliché Roland Jouanisson

4 D'une autre façon l'appartenance terrienne de Pascal a été signifiée par le rattachement du CIBP à ses origines municipales : les congressistes ont été reçus à l'Hôtel de Ville le 9 novembre 1984, par M. le maire-adjoint Pierre Laporte, et le 14 mai 1985 par M. le Maire en personne, Roger Quilliot, universitaire lui-même qui a écrit sur Pascal et Camus. M. Quilliot eut le plaisir de reconnaître parmi nos congressistes un de ses vieux amis japonais.

Mais qu'avons-nous en fait réalisé pour agrandir les voies de «l'accès aux Pensées de Pascal »? Essentiellement nous avons posé et puis défendu une hypothèse. Celle-ci consiste en une prise de conscience d'un état de fait dommageable, constatation qui s'enchaîne avec une espérance. Ce que nous constatons dans la vie culturelle de notre temps et particulièrement dans l'habitus de nos élèves, c'est que l'augmentation à grande vitesse, sur le marché de l'esprit, des connaissances objectives, qu'il s'agisse d'un auteur à comprendre ou de toute autre matière, peut produire un effet d'écrasement, ou au moins d'éparpillement. Or ce qui altère la cohérence de la personne réceptrice diminue la capacité d'écoute. L'espérance qui relève cette réalité négative, c'est que l'effort même qui est tenté pour renouveler la connaissance, produisant un renouvellement d'amitié avec l'objet - en l'occurrence la personnalité de Pascal - augmente du même coup les moyens de la compréhension. Nos fonctions pédagogiques étant d'initiation culturelle, nous sollicitons en nous adressant à Pascal comme à un formateur des formateurs, une sorte de contagion de l'enthousiasme intelligent.

6 Un colloque à visée scientifique et pratique à la fois semble être une nouveauté dans nos mœurs d'enseignants littéraires. Le Courrier du CNRS, dans son supplément au n ${ }^{\circ} 58$, La Vie des Laboratoires (nov.-déc. 1984) l'a fait remarquer. Il était plus facile d'en prendre le risque avec un nombre moyen de participants. Beaucoup d'entre nous se connaissaient déjà, et il s'est instauré, nous semble-t-il, entre tous, un statut de 
confiance amicale. La recherche d'ailleurs était orientée vers les moyens de l'explicitation.

7 Sept exposés d'ouverture à la première session, cinq seulement à la seconde et choisis par les participants de la première, ont ouvert des "chemins" à la discussion fondamentale. Quant aux moyens efficaces de la transmission, c'est dans les six mois d'intervalle entre les deux sessions, que les enseignants les ont expérimentés en équipes, afin de dégager lors de la deuxième session les possibilités d'une pratique. En ce moment toutes ces physionomies de la réflexion sur Pascal sont en train de se fixer sur le papier. L'équipe de rédaction prise du CIBP souhaite que l'année 1985 suffise à les réunir en vue d'un livre équilibré, suggestif et autant que possible séduisant.

8 Mais est-il légitime - et possible - de vouloir séduire avec Pascal ? Faudra-t-il nous inféoder au règne de la concupiscence qu'il a si sévèrement noté ? Notre application sera d'en tirer - comme Pascal lui-même - un meilleur service de la vérité. Certes notre entreprise atteste que nous refusons « le courant de la facilité, du ludique », préférant aux "écrivains flatteurs" les "écrivains formateurs qui font réfléchir", mais tous aussi nous avons découvert que les formateurs "procurent une ouverture durable de l'esprit aident à mieux réfléchir et à mieux vivre. Le plaisir vient au terme d'un travail » (ces expressions sont tirées de l'exposé de Philippe Sellier.) C'est pourquoi nous avons été unanimes à souhaiter que les Actes de nos rencontres, pour avoir une large diffusion, soient édités par le Centre national pédagogique.

9 Si les moyens nous en sont donnés, l'illustration de ces Actes sera belle et nombreuse. Les images en effet sont nécessaires à notre re-construction du milieu pascalien et le premier coup d'œil sur la présente livraison manifeste notre attirance vers les documents visuels. Pour une si discrète publication que d'images! S'il fallait nous en excuser, nous dirions qu'elles sont venues à nous. Cette interprétation par Carlos Pradal du visage de Pascal, nous l'avons rencontrée à l'exposition «Blaise Pascal » de 1983 à Gif-sur-Yvette. (Voir Courrier, n 3, p. 14.) Des intermédiaires amicaux nous ont permis d'obtenir de la propriétaire l'autorisation de la reproduire, et le peintre lui même nous en a confié la photographie en s'expliquant pour nous sur ses intentions. Dirons-nous que la majesté du silence qui enveloppe ce Pascal aux yeux baissés nous a fait penser - avec la différence personnelle d'une douleur humanisée - aux Vierges romanes de notre Auvergne? Dans cette création poétique l'imagination, quoi qu'en ait dit Pascal et conformément à ce qu'il a fait, collabore à la vérité. 


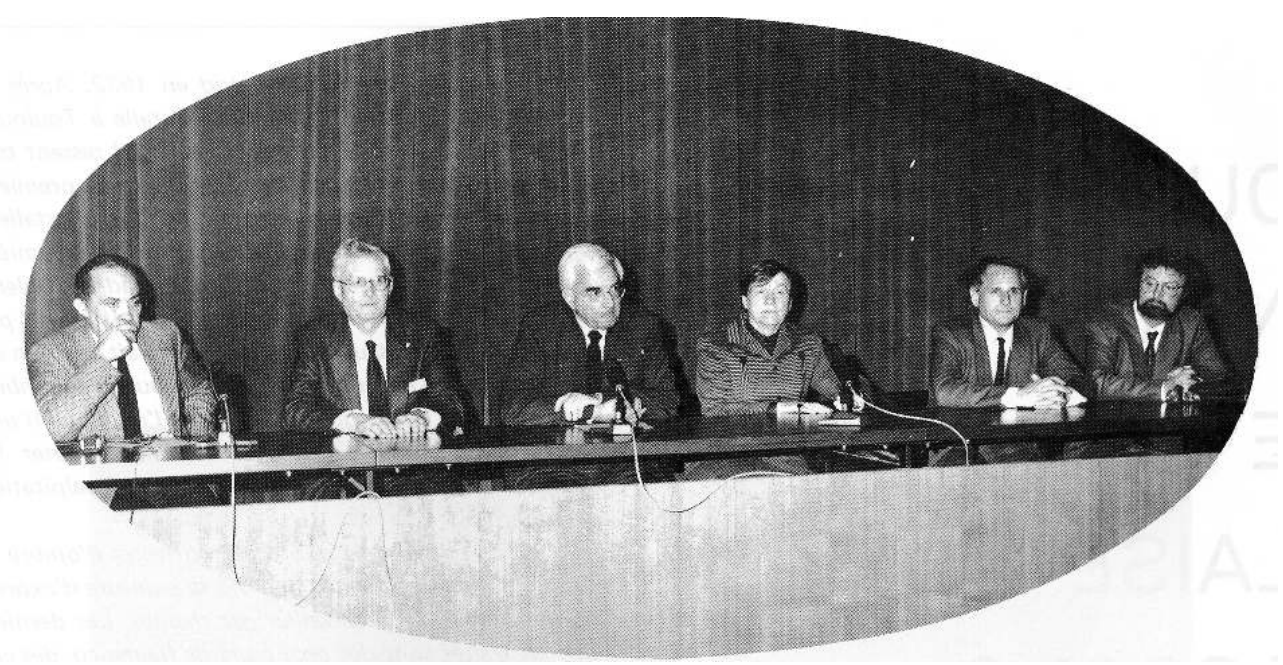

DE gAUCHE À DROITE : M. LE DOYEN J.-CL. PARIENTE, M. JEAN MESNARD, M. LE RECTEUR DISCHAMPS, $M^{M E}$ GOYET, M. L.-P. HENNEQUIN, DIRECTEUR DE LA MISSION ACADÉMIQUE, M. JACQUES MARTY, INSPECTEUR D'ACADÉMIE.

Cliché La Montagne

Et c'est avec un bagage d'imagination encore que le lecteur est invité à refaire à Mirefleurs notre excursion ensoleillée du dimanche 11 octobre 1981. Alors, il faut bien l'avouer, beaucoup d'entre nos congressistes, écarquillant les yeux sur les murs de la maison Domat, n'avaient rien vu. Mais depuis, le temps a réparé les injures du temps, grâce à un propriétaire courageux et fervent de vérité historique. Certes la tour de Mirefleurs ne deviendra pas aussi célèbre que la tour de Montaigne, mais l'article de MM. Ezio Arduini et Yves Morvan la fait désormais entrer en composition dans l'imaginaire dont nous entourons Blaise Pascal lui-même. M. Morvan, professeur d'art et restaurateur de peintures murales, se montre raisonneur prudent autant qu'observateur précis. Il n'affirme pas que ce profil si pascalien numéroté 184 soit de la main même du jurisconsulte dessinateur. Mais enfin il faut bien qu'il soit l'œuvre de quelqu'un de la maison, de la maison qui a reçu, qui a vu Pascal. Que les murs aient des yeux, cela n'est pas interdit dans une maison où les mains des générations successives les couvraient de leurs fantaisies.

11 La mise en lumière de ces crayons sans gloire, témoins d'un plaisir bon enfant, introduit une touche de gaieté dans le tableau des mœurs de la province Auvergne. L'investigation de M. Morvan nous donne à rêver sur les moments de détente qu'a vécus Pascal, en son pays notamment, et il complète - par le contraste - l'austère panégyrique de la famille Domat que nous a laissé, presque un siècle après le temps des visites à Mirefleurs, l'ami de Marguerite Périer, le P. Pierre Guerrier. (Voir « Mémoire sur Domat » dans Mesnard, PASCAL, Euvres complètes, t. I, p. 1158-1164.)

Telle est donc la moisson de réflexions et de figurations qui a été engrangée depuis douze mois dans l'étendue de notre horizon. Notre reconquête historique et spirituelle se fait lentement. Nous pensons que dans cette allure il y a de la sûreté, et de la fécondité, parce que chacune de nos incursions dans le passé et dans les voies de la pensée s'est trouvée introduite par un dévouement amical. C'est cela qui nous porte dans l'avenir. 
13 Nous souhaitons qu'un semblable éveil en chaîne se produise chez les représentants des familles issues de la famille Pascal et qu'ils s'attellent à reconstituer leurs généalogies afin que le CIBP puisse les rassembler. «On recherche la famille ». Cela sera répété à chaque envoi de notre Courrier.

14 Nos associés, nos visiteurs, savent qu'ici on recherche tout ce qui a touché Pascal. Que de pistes entrevues! Mais où nous ne pourrons avancer qu'avec l'aide de ces générosités qui entraînent avec elles les faveurs de la chance.

15 Votre CIBP a toujours besoin de tout le monde.

\section{AUTEUR}

\section{THÉRÈSE GOYET}

Professeur de l'Université II de Clermont-Ferrand 